\title{
Replacement of an Old HPLC Integrator with an Inexpensive Computer and Custom Software
}

BioTechniques 30:1306-1308 (June 2001)

\section{Russell L. Malmberg and Jim Godwin}

University of Georgia, Athens, GA, USA

Our laboratory has an HPLC system originally purchased 15 years ago. The pumps and detectors are still functional, but some of the electronic components that came with the system have begun to fail. In particular, the integrator that came with the HPLC died. The cost of a freestanding integrator or replacing the whole system with a com puter-controlled one seemed high. This note describes the strategy we used to obtain an inexpensive replacement. Our idea was to employ a low-end, underutilized computer as the integrator. The detector output was connected to the computer through an analog/digital computer card and a custom-built amplifier. We wrote a software program to analyze the data that were collected.

The base computer needed is very minimal and usually may be obtained for little cost. We found a four-year-old Pentium ${ }^{\mathbb{R}} \mathrm{I}(133 \mathrm{mHz})$ computer in our university's surplus inventory and are currently using this. We tested an even older 486-based system, and it also worked well.

We obtained the required analog/digital board from Measurement Computing (Middleboro, MA, USA; www. measurement computing. com) in a package known as the Datalab Solution. This contains a CIO-DAS08/JR ISA board, coupled with a version of the software program Labtech Notebook (LabTech, Andover, MA, USA; http:// www.labtech.com/) specifically written for this board. The current price of the combination is $\$ 249$. This particular board will accept input at up to $20 \mathrm{KHz}$ with 12-bit resolution; in other words, it can sample the analog input at up to $1 / 20000$ of a second and can record am plitudes in 1/4096 increments. There are similar analog input boards from other manufacturers and different input boards that will sample at a higher rate and with greater resolution, as needed.

The board we used accepts an ana- $\log$ input on a $+5 /-5 \mathrm{~V}$ scale, but the HPLC Detector has a scale of 0-10 $\mathrm{mV}$ (Beckman Model 157 Fluorescence Detector Instruction Manual, page 1-1; Beckman Model 153 Analytical UV Detector Instrument Manual, page 1-2). We built an amplifier at the University Electronics and Design Shop that scales the detector signal appropriately for a $+5 /-5 \mathrm{~V}$ level into the computer bus card. The interface amplifier unit has output leads that fit the cable leading to

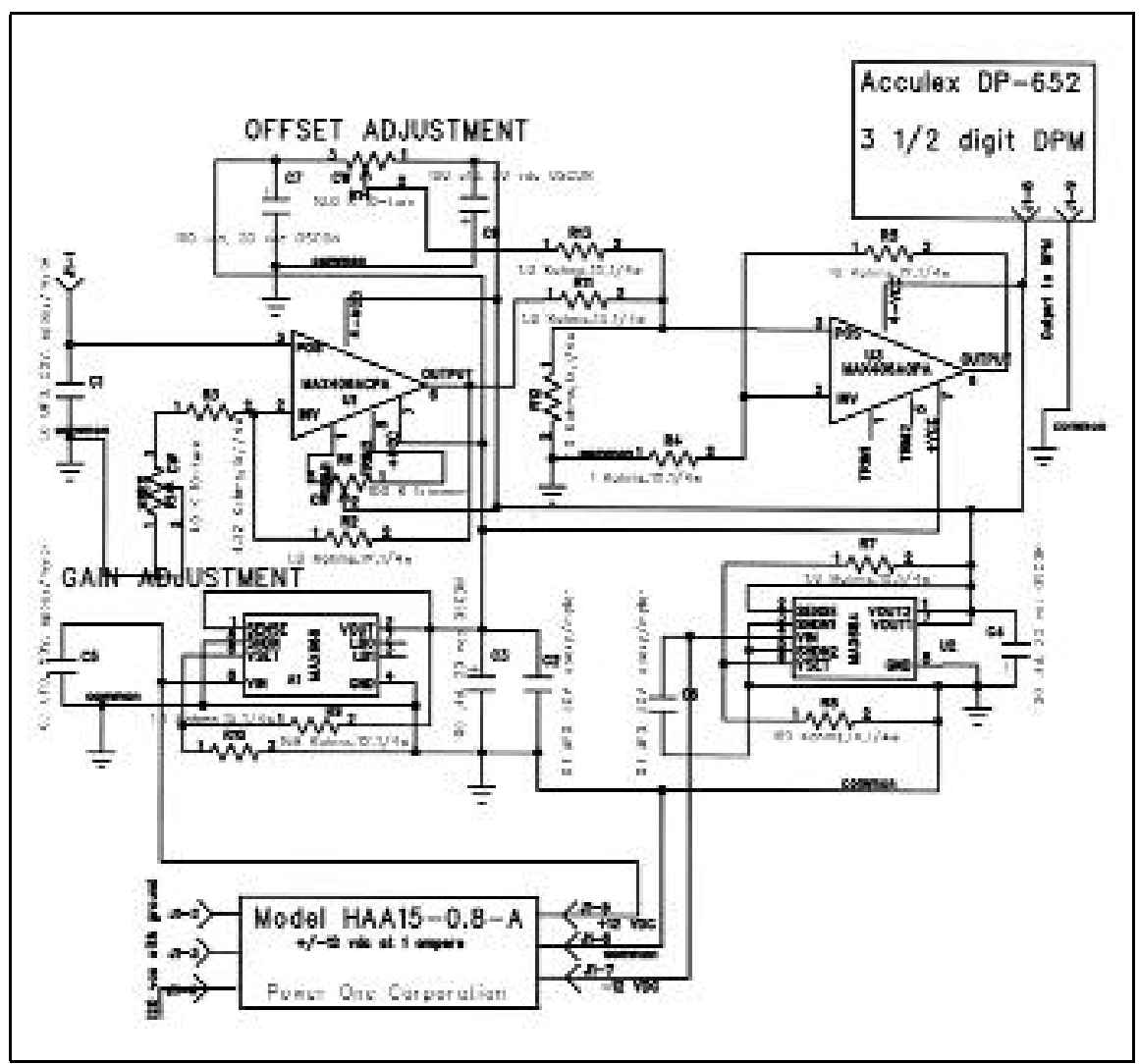

Figure 1. Diagram of the interface circuit that links the detector output to the CIO-DAS08/JR com puter board. 
the computer board, directing the am plifier output to the appropriate pins. It also includes Gain (amplification) and Offset vernier adjustments and 3.5 digit DPM (Digital Panel Meter). These allow one to preview the signal being sent to the computer board, adjust the amplification, and offset appropriately. A schematic diagram of the unit is given in Figure 1. The cost of this amplifier was about $\$ 250$. While this amplifier is stable with little error from signal drift, there are many other variations that also work. The user needs to assure that the amplifier has a relatively high impedance input and low impedance output. This practice will preclude a loading of the detector and erroneous readings.

We used the software that came with the analog input board to create a simple setup in which the detector input into the board is collected at $5 \mathrm{~Hz}$ sam pling; this is displayed immediately on the monitor. The elapsed time is similarly collected at $5 \mathrm{~Hz}$. A keypress function (Alt-1 in our case) is used to trigger collection of the detector output and run time to a file. The user starts this software setup on the computer, and then, when the baseline is stable, the sample is injected, the pump gradient is started, and Alt- 1 is pressed on the computer. The time and detector outputs since the Alt-1 keypress are saved into a file; the Alt-1 keypress combination or simply closing the program are used to terminate the run. The software that came with the analog input board, at least the version we used, directly accesses the hardware; this works fine under Windows $^{\circledR}$ 95/98 but will not work under Windows NT/2000.

While the manufacturer-supplied software can do a variety of data analyses, we were not satisfied with any of its peak area integration results. We therefore wrote a win32 program (for Microsoft Windows 95/98/NT/2000), named Peaks, to integrate the peak areas from the output text file. This program was written in $\mathrm{C}++$ using $\mathrm{Mi}$ crosoft Visual $\mathrm{C}++$. It is based on the CRichEditView class and uses the MFC document/view architecture with

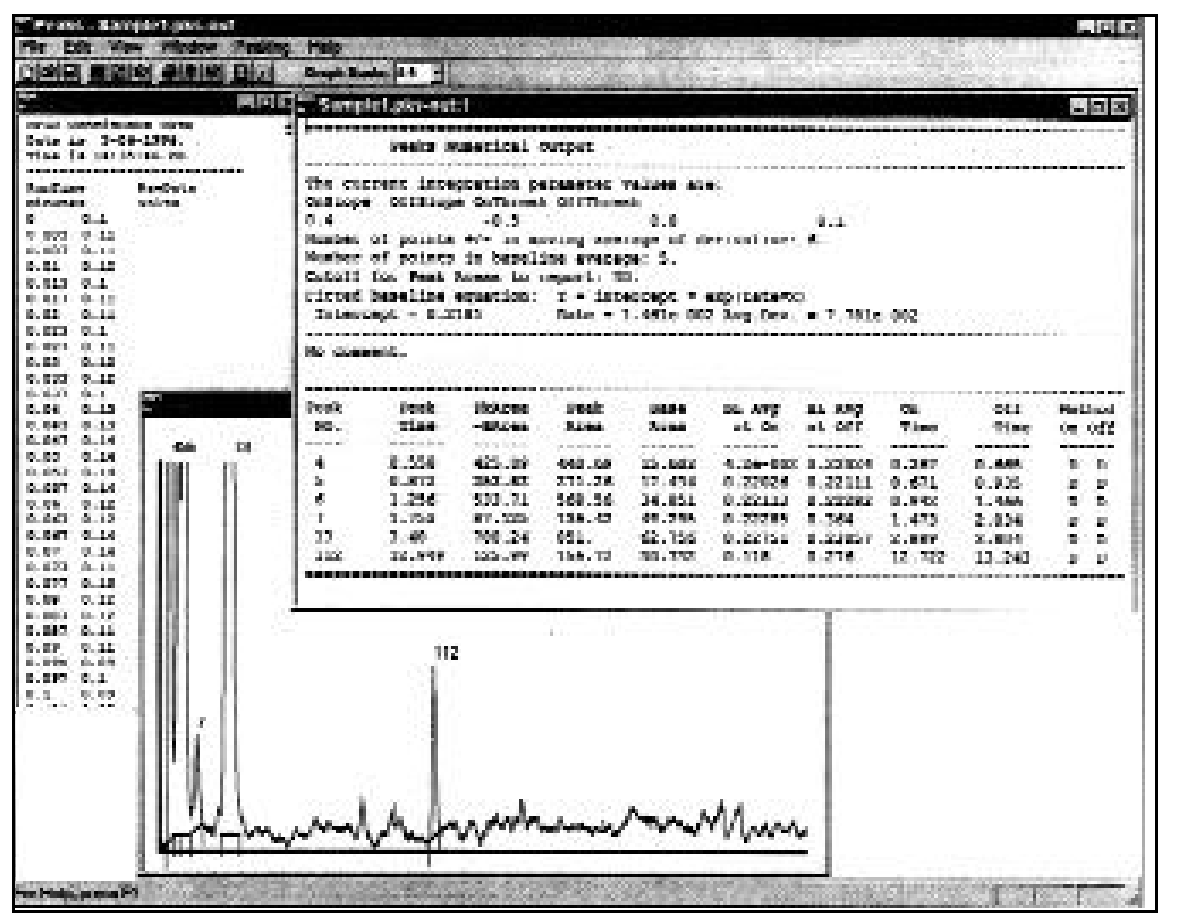

Figure 2. Screenshot of the Peaks program showing input file (upper left), numerical output (upper right), and graphical output (lower center). The peak number in the numerical output corresponds to the peak number in the graphical output. The numerical output includes the peak time, the peak area minus the baseline area, the baseline values at the beginning and ending of the peak, the time at the beginning and ending of the peak, and the method used (slope or threshold value) to detect the beginning and ending of the peak. The heavy blue lines in the graphical output demonstrate the peak beginning and ending, and the baseline. The $\mathbf{I}$ button leads to a dialog that starts the integration process; the $\mathbf{D}$ button leads to a dialog that sets the program parameter defaults; the Graph Scale list box adjusts the size of the output graph window. 
two documents. The input document has a single text view, but the output document has both text and graphical output views. The Peaks program reads the input text file, the user chooses the integration parameters, and then the program produces both a graphical and text output demonstrating the results of its integration analysis. Figure 2 shows a Peaks window screenshot. On the left is the input data; on the upper right is the output text numerical data; on the center bottom is a graphical display of the data. The graphical output puts heavy blue lines to indicate where a given peak integration begins and ends and where the baseline is.

The integration is controlled by a series of parameters accessible through the I (integration) dialog. These allow a user to select what slope values or absolute levels are to be used for each analysis, the minimum area to be reported, and the number of points to use in a moving average. The Peaks program is designed for interactive use to optimize the integration parameters.
Typically, a user tries several sets of values, then examines the graphical outputs; runs can be reanalyzed any number of times to optimize the parameters. When the peak start/stop and baseline appear to be called correctly, then the controlling parameters can be saved in the Peaks.ini file through the D (defaults) dialog. The user can also adjust the minimum peak area to be reported and the zoom of the graphical output to adapt this window for different monitors. The entire dataset in the file can be analyzed, or the user can select just a portion of it for analysis. The program can be adjusted for various anticipated data set sizes in the $\mathbf{D}$ dialog, where one can pick the estimated num ber of data rows and the anticipated number of peaks, both of which should be set larger than the actual data.

The Peaks program does not use sophisticated curve fitting as part of its integration. It simply adds up the areas under the different peak data slices, and, hence, it works well when the number of data points is large. The program does include a variety of tests for split peaks and also includes a baseline exponential curve fitting routine that can provide a correction for a moderately rising or falling baseline. In our laboratory, we record data at $5 \mathrm{~Hz}$ and typically have about 10000 data rows in a run, with 100-300 data slices per peak. The program is able to analyze these data in a few seconds on our 486 or Pentium computers. Modern HPLC column materials or a capillary electrophoresis apparatus may produce peaks that are only a few seconds wide. The acquisition software that comes with the analog input computer board can sample the input as frequently as $1 / 1000$ of a second, and special-purpose software could be written to sam ple the input at the $1 / 20000$ of a second the board is capable of achieving. This hardware/software combination should thus be able to produce accurate representations, with hundreds of data slices, from even the 2-s peak widths that new er laboratory equipment can produce. The Peaks software program can usually generate reliable integration results from as few as 10 data slices per peak, but there is seldom a reason to do this on a routine basis. Instead, the acquisition sampling rate can simply be in- creased to produce a larger number of data slices per second.

Peaks is freely available on the Web (www.botany.uga.edu/malmberg/soft ware.html) and includes a standard Windows help (.hlp) file and setup directions; we tried to make it user friendly. The program stores its settings in the Peaks.ini file in the same directory as the program and, thus, makes no changes to the Windows directory or to the registry. Installing consists of copying the files to an appropriate directory, and uninstalling involves deleting them when done. A number of other laboratories have used the program, with no reports of induced computer crashes or other untoward events. The Peaks program is not specific to this particular computer board or to the Beckman instrumentation, but it can be used to graph and analyze any similar output consisting of two columns of data (corresponding $\mathrm{X}$ and $\mathrm{Y}$ values).

The total cost of our replacement of the HPLC integrator was about $\$ 500$ plus the conversion of a low-end com puter to function as the data collector. The computer is available for other uses when it is not collecting or analyzing the HPLC peaks. It may be possible to similarly replace some components of the HPLC pump controller with an ana$\log$ /digital output computer board, if the electronic signals that control the pumps can be determined. We hope this report and the associated free software will be of use to others who wish to similarly replace a worn-out HPLC integrator.

\section{ACKNOWLEDGMENTS}

We thank Karl Nicholas for programming advice and Mark Watson for extensive testing of the Peaks program. Research in the first author's laboratory is supported by DOE Energy Biosciences grant no. DEFG02-97ER20286

Received 21 November 2000; accepted 5 February 2001.

\author{
Address correspondence to: \\ Dr. Russell L. Malmberg \\ Botany Department \\ University of Georgia \\ Athens, GA 30602-7271, USA \\ e-mail: russell@dogwood.botany.uga.edu
}

